\title{
Estimation of Cardiac Hyperelastic Material Properties from MRI Tissue Tagging and Diffusion Tensor Imaging
}

\author{
Kevin F. Augenstein ${ }^{1}$, Brett R. Cowan ${ }^{2}$, \\ Ian J. LeGrice ${ }^{1}$, and Alistair A. Young ${ }^{1,2}$ \\ ${ }^{1}$ Bioengineering Institute, University of Auckland, New Zealand \\ ${ }^{2}$ Center for Advanced MRI, University of Auckland, New Zealand \\ a. young@auckland.ac.nz
}

\begin{abstract}
The passive material properties of myocardium are important in the understanding of diastolic cardiac dysfunction. We determined hyperelastic myocardial material parameters in four isolated arrested pig hearts undergoing passive inflation of the left ventricle. Using geometry from MRI, recorded boundary conditions, muscle fiber architecture from diffusion tensor imaging, and deformation from tissue tagging, finite element models were constructed to solve the finite elasticity stress estimation problem. The constitutive parameters of a hyperelastic transversely isotropic material law were determined by minimizing the difference between the predicted and imaged deformation field. The optimized parameters were in a similar range as those reported by previous studies, showing increased passive stiffness in the muscle fiber direction. The average RMS error was $0.92 \mathrm{~mm}$, similar to the image resolution of $0.80 \mathrm{~mm}$. Optimization of hyperelastic models of myocardial mechanics can thus be performed to extract meaningful biophysical parameters from MRI data.
\end{abstract}

\section{Introduction}

A better understanding of the motion and mechanical function of the heart in normal and disease states would result in more accurate and effective assessment and diagnoses of cardiac disease in a clinical environment. Current technologies provide a great deal of information about heart function, as there are a large range of imaging modalities (including echocardiography, CT, and MRI) available. However, a lack of applicable and simple models prevents this data from being fully utilized. This is due, in part, to the complex relationship between cardiovascular disease processes and the wide range of observed shapes and motions achieved by the heart. Typically, the data do not densely cover the full region of interest (e.g., MRI provides high resolution data within the imaging plane but relatively low temporal and through-plane resolution). The application of mechanical modeling methods allows interpolation of the sparse data sets over the domain, and characterization of the underlying biophysical phenomena.

In particular, estimation of the compliance of heart tissue is important for the understanding of diastolic dysfunction [1]. Stiffening of the myocardium leads 
to impairment of filling which in turn leads to decreased pump function via the Frank-Starling mechanism. Previous methods proposed to estimate material properties from image data have required limiting assumptions on the form of the material law (often linear elasticity is assumed) or the equations of motion (often small strain theory is imposed) [2, 3, 4, These assumptions are known to be violated in the heart, which undergoes large strains $(20 \%$ shortening and up to $100 \%$ wall thickening) and exhibits nonlinear elastic behavior [5].

We investigated methods for the determination of hyperelastic material parameters from MRI data. In order to fully characterize the boundary conditions applied to the heart, an isolated arrested porcine heart testing protocol was employed. MRI was used to determine the geometry of the heart, the tissue deformation from SPAMM tissue tagging, and the muscle fiber architecture from diffusion tensor imaging. The left ventricle (LV) was cyclically inflated using a computer controlled stepper motor driven pump, with simultaneous LV pressure measurement. The finite elasticity problem was solved using the finite element method, resulting in estimation of stress given a set of material parameters, and the known boundary conditions. The finite elasticity problem was embedded into a parameter optimization loop, which determined the best match between imaged and modeled deformations. This protocol has been previously validated using a silicon gel phantom [6]. Results are presented for four hearts.

\section{Method}

\subsection{Experimental Protocol}

Four pigs were initially anesthetized with zoletil, and maintained with $2.5 \%$ halothane in oxygen. The right femoral artery was cannulated to monitor arterial pressure and a pigtail catheter was inserted into the left ventricle via the right carotid artery. A median and left lateral thoracotomy was performed to expose the heart. The left main coronary artery and right coronary artery were dissected near their origins, and ties were loosely placed for coronary occlusion after arrest. The animal was heparinized (100 IU $\mathrm{kg}^{-1}$ ) via the right carotid artery catheter. A snare was placed around the aorta and the heart was arrested by injection of cold potassium citrate $(60 \mathrm{ml}, 15 \%)$ into the left ventricle via the catheter. Simultaneously, the aortic snare was tightened, thereby perfusing the heart with potassium citrate via the coronary arteries and causing immediate arrest in a relaxed state. The heart was then rapidly removed and rinsed in cold saline, before being perfused with 2,3-butanedione monoaxime (200 ml, $50 \mathrm{mM}$ in saline) through cannulae inserted into the right and left coronary ostia. The coronary arteries were then occluded using the previously placed snares to stop any subsequent flow through the aorta entering the coronary network.

A slit was made in the left atrium to allow access to the mitral valve. A largebore cannula with an annular disc on the end was introduced through the mitral valve, and a thread looped around the cordae tendineae, and tied tight to the cannula above the disc. A second disk was then screwed down on to the valve leaflets clamping them between the upper and lower discs and forming a seal. 
The aortic valve was destroyed and a large diameter cannula introduced in the aorta and sealed with a snare about the aorta. The heart was then suspended in a Plexiglas box (160 mm cube) filled with physiological saline solution, which was clamped into a head coil for imaging. The mitral cannula was attached to a computer controlled reciprocating pump and the aortic cannula attached to the pressure line. Repeatable inflation cycles of approximately $16 \mathrm{ml}$ and a period of $1.2 \mathrm{~s}$ were performed during imaging and pressure measurement [6].

\subsection{Imaging}

SPAMM tagged images were acquired (field of view $200 \mathrm{~mm}, 6 \mathrm{~mm}$ slice thickness, 5 deg flip angle, 128 x 256 matrix, 16 phases, and $5 \mathrm{~mm}$ stripe spacing in a grid pattern) at 6 short axis positions, and at 6 long axis slices at 30 degree increments about the central axis of the left-ventricle. A segmented k-space imaging sequence was used (seven lines per segment, TR 188 ms, TE 4 ms, 2 averages), requiring 38 deformation cycles per image slice to acquire.

In order to obtain an independent measure of the ventricular inflow and out flow during the deformation cycle, phase contrast velocity magnetic resonance images were acquired at the level of the mitral inlet (field of view $150 \mathrm{~mm} \mathrm{x}$ $150 \mathrm{~mm}, 5 \mathrm{~mm}$ slice thickness, 256x256 matrix, 64 phases), prospectively gated on every alternate R-wave so that the entire cycle was acquired. These allowed calculation of volume inflow and outflow through the mitral cannula throughout the deformation cycle.

Diffusion tensor imaging was performed with the heart at zero transmural pressure, in order to obtain information on the tissue micro-structure. A pulsed gradient spin echo diffusion sequence with echo planar imaging and slice readout (two-dimensional multi-slice acquisition, 12 contiguous axial slices, $5 \mathrm{~mm}$ slice thickness, TR $14589.0 \mathrm{~ms}$, TE $100.06 \mathrm{~ms}$, no averages, in plane spatial resolution of $0.82 \mathrm{~mm}$, and slice thickness of $5 \mathrm{~mm}$ ) was used to acquire diffusion-weighted images as described in 7]. Six diffusion gradient direction images (along with a zero weighted image) were acquired per slice, with diffusion weighting b values of $500,1000,1500$ and $2000 \mathrm{~s} \mathrm{~mm}-2$ in each direction, resulting in a data set of 28 images per slice.

\subsection{DTI Analysis}

For each pixel in the region of interest the six independent components of the diffusion tensor $D_{i j}$ and the non-diffusion weighted intensity $(A(0))$ were estimated using Levenberg-Marquardt nonlinear regression. For each pixel, the following objective function was minimized:

$$
\begin{gathered}
\min _{A(0), D_{1 \ldots 3,1 \ldots 3}} \sum_{i=1}^{m} \sum_{j=1}^{n} \frac{1}{\sigma_{j}}\left\|A(0) e^{-b^{i j}: D}-A\left(b^{i j}\right)\right\|_{2}^{2} \\
\text { st } D_{i j}=D_{j i} i, j=1 \ldots 3
\end{gathered}
$$


where $\mathrm{m}$ is the number of diffusion directions $(7), \mathrm{n}$ is the number of gradient magnitudes $(4), b^{i j}$ is the b-matrix with a given diffusion direction and weighting, and $A\left(b^{i j}\right)$ is the image intensity of the pixel. Weights for the 28 residuals (4 magnet strengths times 7 diffusion directions) were set to be $1 / \sigma_{i}$, where $\sigma_{i}$ was the standard deviation of the image intensity in free water for the given diffusion gradient magnitude. The dominant diffusion direction was given by the eigenvector associated with the largest eigenvalue of the diffusion tensor. In the heart, this corresponds to the mean muscle fiber direction in the imaged voxel [8].

\subsection{Finite Element Modeling}

To represent the geometry and the muscle architecture, a finite element model was constructed in 3D rectangular Cartesian coordinates. The geometry and fiber angle field was interpolated using tri-cubic Hermite basis functions, with the element coordinates chosen to lie in the circumferential, longitudinal, and transmural directions respectively. The boundaries of the heart were determined from the untagged images using guide point modeling 9]. Tags were tracked throughout the cycle and 3D displacement fields were reconstructed for each frame using previously described methods [10. The fiber field nodal parameters were optimized to fit the largest eigenvector of the DTI diffusivity tensor.

A transversely-isotropic Fung-type exponential constitutive law was used to characterize the material behavior, as described previously [11, 12, 13, 14]:

$$
W=\frac{1}{2} C\left(e^{b_{1} e_{f f}^{2}+b_{2}\left(e_{r r}^{2}+e_{s s}^{2}+2 e_{s r} e_{r s}\right)+2 b_{3}\left(e_{f s} e_{s f}+e_{f r} e_{r f}\right)-1}\right)
$$

$C, b_{1}, b_{2}$, and $b_{3}$ are material constants and $e_{i j}$ are components of the Lagrangian strain tensor, $e$, referred to the local fibre $(f)$, transmural or radial $(r)$ and crossfibre $(s)$ coordinates. This material law implies material symmetry about the muscle fibre direction, f. Optimization of the material parameters of this law has been performed in several species using various experimental protocols (Tab. 1).

The motions of the basal nodes were constrained to be the same as the imaged motion. All other nodal displacements were unconstrained in the stress solution process. The pressure trace was averaged over every cycle in the imaging sequence, and then pressures for individual image frames were calculated by binning all recorded pressures over the imaging time.

Table 1. Material parameters for the transmural Fung exponential hyperelastic strain energy function, obtained by previous studies

\begin{tabular}{|l|c|c|c|c|c|}
\hline Paper & Species & $\mathrm{C}(\mathrm{kPa})$ & $b_{1}$ & $b_{2}$ & $b_{3}$ \\
\hline Guccione and McCulloch [11] & Canine & 0.88 & 18.5 & 3.58 & 1.63 \\
Okamoto et al. 14] & Canine & 0.3913 & 75.41 & 33.86 & 11.50 \\
& & 1.0173 & 77.49 & 8.016 & 3.122 \\
& & 0.7476 & 39.53 & 6.174 & 5.665 \\
& & 0.3047 & 49.89 & 11.11 & 14.63 \\
& & 0.1008 & 93.03 & 61.61 & 73.10 \\
\hline
\end{tabular}


The material parameters were optimized by solving the finite elasticity equations with a given set of material parameters and boundary conditions. The predicted motion of the tag points was then compared with their imaged motion to calculate an error for each frame:

$$
e=\sum_{i=1}^{N}\left\|x_{i}-x\left(\boldsymbol{\xi}_{i}, \mathbf{C}\right)\right\|_{2}^{2}
$$

where $N$ is the number of data points, $x_{i}$ is the reconstructed deformed data point, and $x\left(\boldsymbol{\xi}_{i}, \mathbf{C}\right)$ is the geometric interpolation function for the deformed geometry, at a given material point, $\mathrm{x}$, and for a given set of material parameters, C.

Sequential Quadratic Programming (SQP) [15] was used to minimize the error with respect to the material parameters. The first derivatives, with respect to the material parameters, were calculated using a finite difference scheme. This involved solving the finite elasticity problem with each perturbed material parameter. In this case, the stress solution was restarted from the unperturbed stress solution in order to reduce solution calculation times. A combined relative and absolute tolerance of 0.001 of the L 2 norm of the change in the material parameters was used as the convergence criterion for the optimization. The Hessian and covariance matrix of the objective function was calculated at the optimum solution, in order to derive information about the correlation between the material parameters.

\subsection{Validation}

The method and apparatus have been previously validated [6]. Briefly, a silicone gel (Sylgard 511 dielectric gel) phantom was constructed in the shape of a cylindrical annulus. The silicon gel was well described by a neo-Hookian material law with a single material parameter $C 1=8.71 \pm 0.06 \mathrm{kPa}$ estimated independently using a rotational shear apparatus. The MRI derived $C_{1}$ parameter was allowed to vary regionally and was estimated as $8.80 \pm 0.86 \mathrm{kPa}$ across the model. Monte Carlo simulations showed that acceptable errors in material parameters (less than $5 \%$ ) were obtained using five frames of the inflation cycle, and noise levels on the order of a pixel (0.8 mm standard deviation).

\section{Results}

The geometry and deformation fields were reconstructed in the finite element model to an RMS error of approximately the pixel size $(0.8 \mathrm{~mm})$. Angular errors in the fiber field ranged from 8 to 13 degrees, comparable to 17 degrees in previous histological studies [16. Figure 1] shows the transmural variation of the fitted fiber field in the model. We found that a finite element mesh with eight elements circumferentially, eight elements longitudinally and two elements transmurally gave converged stress solutions and good numerical convergence of the finite elasticity equations. 


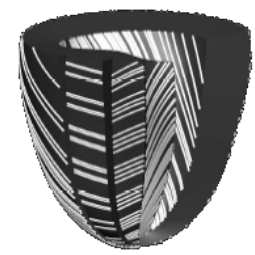

Fig. 1. Transmural fiber field fitted to DTI data at varying depths from epicardial to endocardial surfaces

Table 2. LV pressures at five frames in the inflation cycle for the four experiments

\begin{tabular}{|lllll|}
\hline \multicolumn{4}{|c|}{ Pressures } & $(\mathrm{kPa})$ \\
\hline 0.44 & 0.84 & 1.24 & 1.49 & 1.81 \\
0.54 & 0.78 & 1.07 & 1.40 & 1.51 \\
0.32 & 0.65 & 0.81 & 1.32 & 1.60 \\
0.39 & 0.89 & 1.31 & 1.54 & 2.02 \\
\hline
\end{tabular}

Table 2 shows pressures from five frames of the inflation cycle in the four experiments. These frames were input into the parameter optimization process. Previous validation experiments showed that five frames were sufficient to characterize the material parameters to within $5 \%$ error [6]. Table 3 shows the fitted material parameters for the four hearts used in this study, along with the total error, number of data points and the RMS error. The RMS error is approximately the same size as the pixel size in the original tagged images. A sample correlation matrix is given in Table 4. The condition number for the covariance matrix for the four experiments ranged from 2723 to 6078. Sample short-axis and long-axis images are shown for each of the studies in Fig 2. Superimposed on the images are the model displacements (as crosses). The arrows show the tracked tag points from the reference frame (least pressure) to the frame of highest pressure.

\section{Discussion}

Finite element models of geometry and micro-structure were constructed from MRI derived data. These models, together with tracked material points and

Table 3. Optimal material parameters and RMS errors for the four experiments

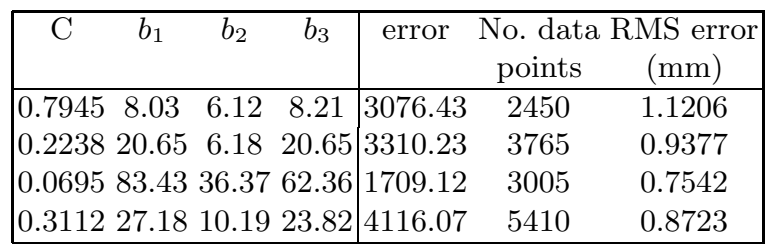


Table 4. Typical correlation matrix at the optimal solution

\begin{tabular}{|c|c|c|c|c|}
\hline & \multicolumn{4}{|c|}{ Correlation } \\
\hline & $\mathrm{C}$ & $b_{1}$ & $b_{2}$ & $b_{3}$ \\
$\mathrm{C}$ & 1.0000 & 0.2062 & -0.6408 & -0.1129 \\
$b_{1}$ & & 1.0000 & 0.0313 & 0.0618 \\
$b_{2}$ & & & 1.0000 & -0.5429 \\
$b_{3}$ & & & & 1.0000 \\
\hline
\end{tabular}
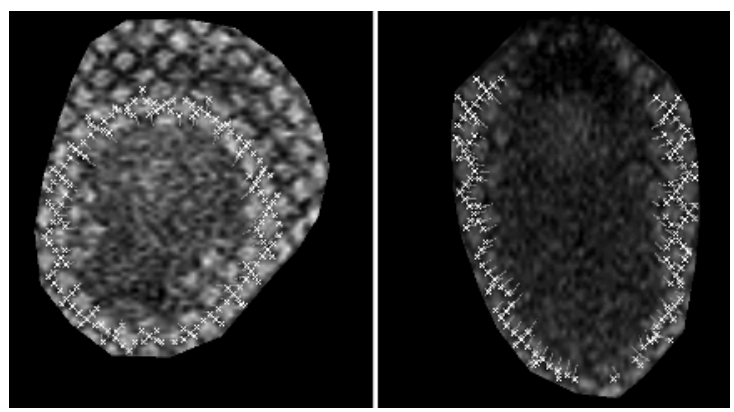

Fig. 2. Short and long axis model displacements (arrows) and predicted tag stripe locations (white points)

recorded pressures, were used to estimate material parameters. One novel aspect of this experimental protocol was that the papillary muscles were left attached to the mitral valve leaflets which were tied. However, in the model, the papillary muscles were excluded and their effects on the mechanical response is yet to be determined. The fitted parameters differed between experiments, but fell within the range previously obtained in the literature (Table 1).

The large condition number of the Hessian indicates that parameters of this law are not well defined by the experimental data, although the fitted parameters were capable of reconstructing the tracked material points with an RMS of $0.92 \mathrm{~mm}$, approximately equal to the image resolution of $0.8 \mathrm{~mm}$. The choice of the appropriate material law formulation for heart myocardium, including allowing for possible regional variations of parameters, is currently an open research topic. [17] have proposed alternative formulations based on strain attributes which are chosen to minimize the correlation between terms in the material law. Myocardial material laws based on this formulation show promise for material parameter estimation from magnetic resonance displacement data, by reducing the correlation between fitted parameters (as found in the current study) and thereby improving the accuracy of the parameter estimation.

In order to extend this work to characterize the material properties of the heart in-vivo, the left ventricle filling pressure and other boundary conditions must be non-invasively determined. Computational fluid dynamics may be used to determine noninvasive pressure gradients in late filling, which may then be combined with strain measurements [3]. 


\section{References}

1. Mandinov, L., Eberli, F.R., Seiler, C., Hess, O.M.: Diastolic heart failure. Cardiovasc. Res. 45 (2000) 813-825

2. Hu, Z., Metaxas, D., Axel, L.: In vivo strain and stress estimation of the heart left and right ventricles from mri images. Medical Image Analysis 7 (2003) 435-444

3. Wen, H., Bennett, E., Epstein, N., Plehn, J.: Magnetic resonance imaging assessment of myocardial elastic modulus and viscosity using displacement imaging and phase-contrast velocity mapping. Magn Reson Med 54 (2005) 538-548

4. Sermesant, M., Rhode, K., Sanchez-Ortiz, G., Camara, O., Andriantsimiavona, R., Hegde, S., Rueckert, D., Lambiase, P., Bucknall, C., Rosenthal, E., Delingette, H., Ayache, N., Razavi, R.: Simulation of caridac pathologies using an electromechanical biuventricular model and xmr interventional imaging. Medical Image Analysis 9 (2005) 467-480

5. Yin, F.: Ventricular wall stress. Circulation Research 49 (1981) 829-842

6. Augenstein, K., Cowan, B., LeGrice, I., Nielsen, P., Young, A.: Method and apparatus for soft tissue material parameter estimation using magnetic resonance imaging. J Biomech Eng. 127 (2005) 148-157

7. Basser, P., Pierpaoli, C.: A simplified method to measure the diffusion tensor from seven mr images. Magn Reson Med 39 (1998) 928-934

8. Garrido, L., Wedeen, V., Kwong, K., Spencer, U., Kantor, H.: Anisotropy of water diffusion in the myocardium of the rat. Circ Res. 74 (1994) 789-93

9. Young, A., Cowan, B., Thrupp, S., Hedley, W., Dell'Italia, L.: Left ventricular mass and volume: fast calculation with guide-point modeling on MR images. Radiology 216 (2000) 597-602

10. Young, A., Kraitchman, D., Dougherty, L., Axel, L.: Tracking and finite element analysis of stripe deformation in magnetic resonance tagging. IEEE Trans Medical Imaging 14 (1995) 413-421

11. Guccione, J.M., McCulloch, A.D., Waldman, L.K.: Passive material properties of intact ventricular myocardium determined from a cylindrical model. ASME Journal of Biomechanical Engineering 113 (1991) 42-55

12. Guccione, J.M., McCulloch, A.D.: Finite element modeling of ventricular mechanics. In Glass, L., Hunter, P., McCulloch, A.D., eds.: Theory of Heart - Biomechanics, Biophysics, and Nonlinear Dynamics of Cardiac Function, Springer-Verlag, New York (1991) 121-44

13. Emery, J.L., Omens, J.H., McCulloch, A.D.: Strain softening in rat left ventricular myocardium. ASME Journal of Biomechanical Engineering 119 (1997) 6-12

14. Okamoto, R.J., Moulton, M.J., Peterson, S.J., Li, D., Pasque, M.K., Guccione, J.M.: Epicardial suction: A new approach to mechanical testing of the passive ventricular wall. Journal of Biomechanical Engineering 1122 (2000) 479-487

15. Gill, P.E., Murray, W., Wright, M.H. In: Practical Optimization, (Academic Press, New York)

16. P. M. F. Nielsen, I. J. Grice, B.H.S., Hunter, P.J.: Mathematical model of the geometry and fibrous structure of the heart. American Journal of Physiology 260 (1991) H1365-H1378

17. Criscione, J.C., McCulloch, A.D., Hunter, W.C.: Constitutive framework optimized for myocardium and other high-strain, laminar materials with one fiber family. Journal of the Mechanics and Physics of Solids 50 (2002) 1681-702 